\title{
THE ASYMPTOTIC DISTRIBUTIONS OF STATISTICS BASED ON LOGARITHMS OF SPACINGS
}

1. Introduction and preliminaries. Let $X_{1}, \ldots, X_{n}$ be a sample from a distribution on the interval $[0,1]$. We denote by $0=X_{0: n} \leq X_{1: n} \leq$ $\ldots \leq X_{n: n} \leq X_{n+1: n}=1$ the order statistics from the sample. The purpose of this paper is to find the asymptotic distributions of the statistics $\sum_{i=0}^{n} \log \left(X_{i+1: n}-X_{i: n}\right)$ and $\sum_{i=0}^{n}\left(X_{i+1: n}-X_{i: n}\right) \log \left(X_{i+1: n}-X_{i: n}\right)$ in the case when the distribution of the sample has a density which is a step function on $[0,1]$. The asymptotic distributions of the above statistics in the case of the uniform distribution on $[0,1]$ were found by Darling [1] and Gebert and Kale [2], respectively. The problem of finding asymptotic distributions of some other statistics based on distributions with densities in the form of step functions was considered by Weiss [3], [4].

Let $k \geq 1$ be a fixed integer and let $0=x_{0}<x_{1}<\ldots<x_{k}=1$ be fixed real numbers. Let us introduce the following notation:

$$
\begin{gathered}
I_{0}=[0,1], \quad I_{1}=\left[x_{0}, x_{1}\right), \quad \ldots, \quad I_{k-1}=\left[x_{k-2}, x_{k-1}\right), \quad I_{k}=\left[x_{k-1}, x_{k}\right], \\
\left|I_{i}\right|=x_{i}-x_{i-1}, \quad i=1, \ldots, k .
\end{gathered}
$$

Let $f_{i}>0, i=1, \ldots, k$, be fixed numbers such that $\sum_{i=1}^{k} f_{i}\left|I_{i}\right|=1$. These numbers together with the intervals $I_{i}$ define a probability density $f$ :

$$
f(x)=\sum_{i=1}^{k} f_{i} \mathbf{1}_{I_{i}}(x) .
$$

Define $p_{i}=f_{i}\left|I_{i}\right|$. We have $\sum p_{i}=1$ and hence there exist numbers $0=$ $x_{0}^{\prime}<x_{1}^{\prime}<\ldots<x_{k}^{\prime}=1$ such that the intervals $I_{i}^{\prime}$, defined similarly to $I_{i}$, have lengths $p_{i}$. It is well known that there exists a probability space $(\Omega, \mathcal{F}, P)$, on which we can define a vector of random elements 


$$
\left(\underline{U}, \underline{Y}_{1}, \ldots, \underline{Y}_{k}\right): \Omega \rightarrow\left(\mathbb{R}^{\infty}\right)^{k+1}
$$

with the following properties:

(a) the coordinates of this vector are stochastically independent,

(b) $\underline{U}=\left(U_{1}, U_{2}, \ldots\right)$ is a sequence of independent random variables uniformly distributed on $I_{0}$,

(c) for $i=1, \ldots, k, \underline{Y}_{i}=\left(Y_{i, 1}, Y_{i, 2}, \ldots\right)$ are sequences of independent random variables with uniform distribution on $I_{i}$.

We can now define a sequence $\underline{Z}=\left(Z_{1}, Z_{2}, \ldots\right)$ of independent random variables with density $f$ :

$$
Z_{n}=\sum_{i=1}^{k} \mathbf{1}_{I_{i}^{\prime}}\left(U_{n}\right) Y_{i, n}, \quad n \geq 1 .
$$

The independence of the variables $Z_{n}$ results from the properties of the vector (2). We check that they have density $f$. Let $A \subset I_{0}$ be a Borel set and let $\lambda$ be the Lebesgue measure. Then we have

$$
\begin{aligned}
P\left(Z_{n} \in A\right) & =\sum_{i=1}^{k} P\left(Z_{n} \in A \cap I_{i}\right)=\sum_{i=1}^{k} P\left(U_{n} \in I_{i}^{\prime}\right) P\left(Y_{i, n} \in A \cap I_{i}\right) \\
& =\sum_{i=1}^{k} f_{i}\left|I_{i}\right| \frac{\lambda\left(A \cap I_{i}\right)}{\left|I_{i}\right|}=\int_{A} f(x) d \lambda(x) .
\end{aligned}
$$

Now denote by $N_{i, n}$ the number of random variables $Z_{1}, \ldots, Z_{n}$ taking values in the interval $I_{i}$, that is,

$$
N_{i, n}=\sum_{j=1}^{n} \mathbf{1}_{I_{i}}\left(Z_{j}\right) .
$$

Since $Z_{j} \in I_{i}$ if and only if $U_{j} \in I_{i}^{\prime}$, we have

$$
N_{i, n}=\sum_{j=1}^{n} \mathbf{1}_{I_{i}^{\prime}}\left(U_{j}\right) .
$$

It follows that the sequence $\left(N_{1, n}, \ldots, N_{k, n}\right)$ is independent of $\left(\underline{Y}_{1}, \ldots, \underline{Y}_{k}\right)$. For simplicity of notation we now introduce two real functions defined on $\mathbb{N} \times I_{0}^{\infty}$ as follows:

$$
\begin{aligned}
\phi(n, \underline{y}) & =\sum_{j=0}^{n} \log \left(y_{j+1: n}-y_{j: n}\right), \\
\phi^{*}(n, \underline{y}) & =\sum_{j=0}^{n}\left(y_{j+1: n}-y_{j: n}\right) \log \left(y_{j+1: n}-y_{j: n}\right),
\end{aligned}
$$


where $n \geq 1, \underline{y}=\left(y_{1}, y_{2}, \ldots\right) \in I_{0}^{\infty}$. The Darling and the Gebert-Kale statistics based on a sample from the uniform distribution on $I_{0}$ can now be written as $\phi(n, \underline{U})$ and $\phi^{*}(n, \underline{U})$, where $\underline{U}$ is the sequence (2).

2. Darling statistic. The asymptotic distribution of the Darling statistic in the case of the uniform distribution on $I_{0}$ is given by the following theorem:

LEMma 1 (Darling [2]). If $\underline{U}=\left(U_{1}, U_{2}, \ldots\right)$ is a sequence of independent random variables with uniform distribution on $[0,1]$, then

$$
\frac{\phi(n, \underline{U})+n(\log n+\gamma)}{\sqrt{\pi^{2} / 6-1} \sqrt{n}} \stackrel{d}{\rightarrow} N(0,1),
$$

where $\gamma=0.5772 \ldots$ is Euler's constant.

Using Lemma 1 we find the limiting distribution of the statistic $\phi$ when the distribution of the sample variables is given by (1):

THEOREM 1. If $\underline{Z}=\left(Z_{1}, Z_{2}, \ldots\right)$ is a sequence of independent random variables distributed according to (1) then

$$
\frac{\phi(n, \underline{Z})+n\left\{E\left[\log \left(n f\left(Z_{1}\right)\right)\right]+\gamma\right\}}{\sqrt{\pi^{2} / 6-1+\operatorname{Var}\left[\log f\left(Z_{1}\right)\right]} \sqrt{n}} \stackrel{d}{\rightarrow} N(0,1) .
$$

To prove Theorem 1 we need some lemmas.

Lemma 2. Let $X, X_{n}, n \geq 1$, be random variables such that $X_{n} \stackrel{P .1}{\longrightarrow} 0$, $\sqrt{n} X_{n} \stackrel{d}{\rightarrow} X$. Then

$$
\sqrt{n}\left|\log \left(1+X_{n}\right)-X_{n}\right| \stackrel{P}{\rightarrow} 0 .
$$

P r o of. Using the Taylor expansion it is easy to show that $|\log (1+x)-x|$ $\leq x^{2}$ for $|x| \leq 1 / 2$. Consider the set $B_{n}=\bigcap_{j=n}^{\infty}\left\{\left|X_{j}\right| \leq 1 / 2\right\}$. Since $X_{n} \stackrel{P .1}{\longrightarrow} 0, \lim P\left(B_{n}\right)=1$. The assumption that $X_{n} \stackrel{P .1}{\longrightarrow} 0$, together with $\sqrt{n} X_{n} \stackrel{d}{\rightarrow} X$, gives $\sqrt{n} X_{n}^{2} \stackrel{P}{\rightarrow} 0$. We conclude that for arbitrary $\varepsilon, \eta>0$ there exists $j_{0}$ such that $P\left(\Omega \backslash B_{j_{0}}\right)<\eta$ and $P\left(\sqrt{n} X_{n}^{2} \geq \varepsilon\right) \leq \eta$ for $n \geq j_{0}$. Hence

$$
\begin{aligned}
P\left(\sqrt{n}\left|\log \left(1+X_{n}\right)-X_{n}\right| \geq \varepsilon\right) & \\
& \leq P\left(\Omega \backslash B_{j_{0}}\right)+P\left(B_{j_{0}}, \sqrt{n}\left|\log \left(1+X_{n}\right)-X_{n}\right| \geq \varepsilon\right) \\
& \leq \eta+P\left(B_{j_{0}}, \sqrt{n} X_{n}^{2} \geq \varepsilon\right) \leq 2 \eta \quad \text { for } n \geq j_{0} .
\end{aligned}
$$

This proves (10).

LeMmA 3. For arbitrary $i=1, \ldots, k$ and $\varepsilon>0$ we have

$$
\frac{1}{\sqrt{n}}\left|\log \left(\min _{1 \leq j \leq N_{i, n}} Y_{i, j}-x_{i-1}\right)\right| \stackrel{P}{\rightarrow} 0
$$




$$
\frac{1}{\sqrt{n}}\left|\log \left(x_{1}-\max _{1 \leq j \leq N_{i, n}} Y_{i, j}\right)\right| \stackrel{P}{\rightarrow} 0 .
$$

Proof. The random variable $N_{i, n}$ is independent of the sequence $\underline{Y}_{i}$, and $N_{i, n} \stackrel{P .1}{\longrightarrow} \infty$, so it suffices to show that

$$
\frac{1}{\sqrt{n}}\left|\log \left(\min _{j \leq j \leq n} Y_{i, j}-x_{i-1}\right)\right| \stackrel{P}{\rightarrow} 0,
$$

and similarly in the case of (12). We have

$$
\begin{aligned}
P\left(\frac{1}{\sqrt{n}}\left|\log \left(\min _{1 \leq j \leq n} Y_{i, j}-x_{i-1}\right)\right| \geq \varepsilon\right) & =P\left(\min _{1 \leq j \leq n} Y_{i, j}-x_{i, j} \leq \exp (-\varepsilon \sqrt{n})\right) \\
& =1-\left(1-\left|I_{i}\right|^{-1} \exp (-\varepsilon \sqrt{n})\right)^{n} .
\end{aligned}
$$

We now prove that $\left(1-\left|I_{i}\right|^{-1} \exp (-\varepsilon \sqrt{n})\right)^{n} \rightarrow 1$. Take arbitrary $\eta>0$ and $\delta>0$ such that $\exp (-\delta) \geq 1-\eta$. For sufficiently large $n$,

$$
1 \geq\left(1-\left|I_{i}\right|^{-1} \exp (-\varepsilon \sqrt{n})\right)^{n} \geq(1-\delta / n)^{n} \geq \exp (-\delta)-\eta \geq 1-2 \eta .
$$

This ends the proof of Lemma 3.

LEMma 4. Let $X_{i, n}, i=1, \ldots, k, n \geq 0$, be the random variables defined as follows:

$$
X_{i, n}= \begin{cases}0 & \text { for } n=0, \\ \frac{1}{\sqrt{\pi^{2} / 6-1} \sqrt{n}}\left[\phi\left(n, \underline{Y}_{i}\right)+n\left(\log \frac{n}{\left|I_{i}\right|}+\gamma\right)\right] & \text { for } n \geq 1 .\end{cases}
$$

Then

$$
\begin{aligned}
\left(X_{i, N_{i, n}}, i=1, \ldots, k, \frac{N_{i, n}-n p_{i}}{\sqrt{n}}, i\right. & =1, \ldots, k) \\
& \stackrel{d}{\rightarrow}\left(X_{1}, \ldots, X_{k}, W_{1}, \ldots, W_{k}\right),
\end{aligned}
$$

where the $X_{i}$ are independent and normally $N(0,1)$ distributed random variables, the vector $\left(W_{1}, \ldots, W_{k}\right)$ is independent of $\left(X_{1}, \ldots, X_{k}\right)$ and has the multivariable normal distribution $N(0, \Sigma)$, where $\Sigma=\left[\sigma_{i, j}\right]$ and

$$
\sigma_{i, j}= \begin{cases}-p_{i} p_{j} & \text { for } i \neq j, \\ p_{i}-p_{i}^{2} & \text { for } i=j\end{cases}
$$

$i, j=1, \ldots, k$.

Proof. We first show that $\left(X_{1, n}, \ldots, X_{k, n}\right) \stackrel{d}{\rightarrow}\left(X_{1}, \ldots, X_{k}\right)$. It is obvious that spacings from the uniform distribution on a certain interval divided by the length of this interval have the same distribution as spacings from the uniform distribution on $[0,1]$. Thus

$$
\frac{1}{\sqrt{\pi^{2} / 6-1} \sqrt{n}}\left[\phi\left(n, \underline{Y}_{i} /\left|I_{i}\right|\right)+n(\log n+\gamma)\right] \stackrel{d}{\rightarrow} X_{i} .
$$


Moreover,

$$
\left.\phi\left(n, \underline{Y}_{i} /\left|I_{i}\right|\right)+n(\log n+\gamma)=\phi\left(n, \underline{Y}_{i}\right)+n \log \left(n /\left|I_{i}\right|\right)+\gamma\right)-\log \left|I_{i}\right| .
$$

Since $\log \left(\left|I_{i}\right| / \sqrt{n}\right) \rightarrow 0$, we have $X_{i, n} \stackrel{d}{\rightarrow} X_{i}$. Because $X_{i}$ are independent, we have also

$$
\left(X_{1, n}, \ldots, X_{k, n}\right) \stackrel{d}{\rightarrow}\left(X_{1}, \ldots, X_{k}\right)
$$

Now we show that

$$
\left(\frac{N_{1, n}-n p_{1}}{\sqrt{n}}, \ldots, \frac{N_{k, n}-n p_{k}}{\sqrt{n}}\right) \stackrel{d}{\rightarrow}\left(W_{1}, \ldots, W_{k}\right) .
$$

This follows from the central limit theorem because $N_{i, n}=\sum_{j=1}^{n} \mathbf{1}_{I_{1}^{\prime}}\left(U_{j}\right)$, $E\left[\mathbf{1}_{I_{i}^{\prime}}\left(U_{1}\right)\right]=p_{i}$ and $\operatorname{Cov}\left[\mathbf{1}_{I_{i}^{\prime}}\left(U_{1}\right), \mathbf{1}_{I_{j}^{\prime}}\left(U_{1}\right)\right]=\sigma_{i, j}$. Since the sequence $N_{i, n}$ is independent of the sequence $\underline{Y}_{i},(14)$ and (15) together give (13).

Proof of Theorem 1. Consider the sequence $Z_{\alpha_{i, 1}}, Z_{\alpha_{i, 2}}, \ldots$ of those successive random variables $Z_{n}$ whose values belong to the interval $I_{i}$. The sequence $\alpha_{i, n}, n \geq 1$, is determined by the sequence $\underline{U}$, so it is independent of $\underline{Y}_{i}$. Since $Z_{\alpha_{i, n}}=Y_{i, \alpha_{i, n}}$, the sequence $Z_{\alpha_{i, n}}$ has the same probability distribution as $\underline{Y}_{i}$. It can be shown similarly that the joint distribution of the vector $\left(Z_{\alpha_{i, n}}, n \geq 1, \ldots, Z_{\alpha_{k, n}}, n \geq 1\right)$ is the same as that of $\left(\underline{Y}_{1}, \ldots, \underline{Y}_{k}\right)$. It follows from the above remarks and from Lemma 3 that the statistic $\phi(n, \underline{Z})$ has asymptotically the same distribution as $\sum_{i=1}^{k} \phi\left(N_{i, n}, \underline{Y}_{i}\right)$.

To prove Theorem 1 it is now enough to show that

$$
\frac{\sum_{i=1}^{k} \phi\left(N_{i, n}, \underline{Y}_{i}\right)+n\left(E\left[\log \left(n f\left(Z_{1}\right)\right)\right]+\gamma\right)}{\sqrt{n} \sqrt{\pi^{2} / 6-1+\operatorname{Var}\left[\log f\left(Z_{1}\right)\right]}} \stackrel{d}{\rightarrow} N(0,1) .
$$

Set $C=\sqrt{\pi^{2} / 6-1}$. Since $\sum_{i=1}^{k} N_{i, n}=n$, after elementary calculations we obtain

$$
\begin{aligned}
& \frac{1}{C \sqrt{n}}\left[\sum_{i=1}^{k} \phi\left(N_{i, n}, \underline{Y}_{i}\right)+n\left(E\left[\log \left(n F\left(Z_{1}\right)\right)\right]+\gamma\right)\right] \\
& =\frac{1}{C \sqrt{n}}\left[\sum_{i=1}^{k} \phi\left(N_{i, n}, \underline{Y}_{i}\right)+N_{i, n}\left(\log \frac{N_{i, n}}{\left|I_{i}\right|}+\gamma\right)\right] \\
& \quad-\frac{1}{C \sqrt{n}} \sum_{i=1}^{k-1}\left(N_{i, n}-n p_{i}\right) \log \frac{N_{i, n}}{N_{k, n}}+\frac{1}{C \sqrt{n}} \sum_{i=1}^{k}\left(N_{i, n}-n p_{i}\right) \log \left|I_{i}\right| \\
& \quad-\frac{1}{C \sqrt{n}} \sum_{i=1}^{k} n p_{i} \log \left(\frac{N_{i, n}}{n p_{i}}\right) .
\end{aligned}
$$


We first show that

$$
\frac{1}{\sqrt{n}} \sum_{i=1}^{k} n p_{i} \log \left(\frac{N_{i, n}}{n p_{i}}\right) \stackrel{P}{\rightarrow} 0 .
$$

Define $\alpha(x)=\log (1+x)-x$. We have

$$
\begin{aligned}
\sum_{i=1}^{k} p_{i} \log \left(\frac{N_{i, n}}{n p_{i}}\right) & =\sum_{i=1}^{k} p_{i} \frac{N_{i, n}-n p_{i}}{n p_{i}}+\sum_{i=1}^{k} p_{i} \alpha\left(\frac{N_{i, n}-n p_{i}}{n p_{i}}\right) \\
& =\sum_{i=1}^{k} p_{i} \alpha\left(\frac{N_{i, n}-n p_{i}}{n p_{i}}\right) .
\end{aligned}
$$

Lemma 2 shows that

and this implies (18).

$$
\sqrt{n} \alpha\left(\frac{N_{i, n}-n p_{i}}{n p_{i}}\right) \stackrel{P}{\rightarrow} 0
$$

It follows from (17), (18) and Lemma 4 that

$$
\begin{aligned}
& \frac{1}{C \sqrt{n}}\left[\sum_{i=1}^{k} \phi\left(N_{i, n}, \underline{Y}_{i}\right)+n\left(E\left[\log \left(n f\left(Z_{1}\right)\right)\right]+\gamma\right)\right] \\
& \stackrel{d}{\rightarrow} \sum_{i=1}^{k} \sqrt{p_{i}} X_{i}+\frac{1}{C} \sum_{i=1}^{k} W_{i} \log \left|I_{i}\right|-\frac{1}{C} \sum_{i=1}^{k-1} W_{i} \log \frac{p_{i}}{p_{k}},
\end{aligned}
$$

where $X_{i}, W_{i}$ are the same as in Lemma 4. Since $\sum_{i=1}^{k} p_{i}=1$ and $\sum_{i=1}^{k} W_{i}=$ 0 , we have

$$
\sum_{i=1}^{k} W_{i} \log \left|I_{i}\right|-\sum_{i=1}^{k-1} W_{i} \log \frac{p_{i}}{p_{k}}=\sum_{i=1}^{k} W_{i} \log \frac{\left|I_{i}\right|}{p_{i}} .
$$

The variance of the sum of the coordinates of an arbitrary random vector is equal to the sum of the elements of the covariance matrix of this vector. Hence

$$
\begin{aligned}
\operatorname{Var}\left[\sum_{i=1}^{k} W_{i} \log \frac{\left|I_{i}\right|}{p_{i}}\right]=\sum_{i=1}^{k} \sum_{j=1}^{k}\left(\log \frac{\left|I_{i}\right|}{p_{i}} \log \frac{\left|I_{j}\right|}{p_{j}}\right) \sigma_{i, j} \\
=\sum_{i=1}^{k} p_{i} \log ^{2} \frac{\left|I_{i}\right|}{p_{i}}-\left(\sum_{i=1}^{k} p_{i} \log \frac{\left|I_{i}\right|}{p_{i}}\right)^{2}=\operatorname{Var}\left[\log f\left(Z_{1}\right)\right] .
\end{aligned}
$$

Now (19) shows that

$$
\frac{\sum_{i=1}^{k} \phi\left(N_{i, n}, \underline{Y}_{i}\right)+n\left(E\left[\log \left(n f\left(Z_{1}\right)\right)\right]+\gamma\right)}{\sqrt{n} C \sqrt{1+\left(1 / C^{2}\right) \operatorname{Var}\left[\log f\left(Z_{1}\right)\right]}} \stackrel{d}{\rightarrow} N(0,1) .
$$

This ends the proof of Theorem 1 . 
3. Gebert-Kale statistic. In this section we prove an analogue of Theorem 1 concerning the Gebert-Kale statistic. Our considerations are based on the following theorem which gives the asymptotic distribution of the Gebert-Kale statistic in the case of the uniform distribution on $[0,1]$.

LEMMA 5 (Gebert and Kale [2]). If $\underline{U}=\left(U_{1}, U_{2}, \ldots\right)$ is a sequence of independent random variables uniformly distributed on $[0,1]$ then

$$
\frac{\sqrt{n}}{\sqrt{\pi^{2} / 3-3}}\left(\phi^{*}(n, \underline{U})+\log n-(1-\gamma)\right) \stackrel{d}{\rightarrow} N(0,1) .
$$

The asymptotic distribution of the Gebert-Kale statistic when the underlying distribution has density (1) is given by the following theorem.

THEOREM 2. If $\underline{Z}=\left(Z_{1}, Z_{2}, \ldots\right)$ is a sequence of independent random variables with density (1) then

$$
\frac{\sqrt{n}\left(\phi^{*}(n, \underline{Z})+E\left[\frac{\log \left(n f\left(Z_{1}\right)\right)}{f\left(Z_{1}\right)}\right]-(1-\gamma)\right)}{\sqrt{\left(\frac{\pi^{2}}{3}-2\right) \operatorname{Var}\left[\frac{1}{f\left(Z_{1}\right)}\right]+\left(\frac{\pi^{2}}{3}-3\right)}} \stackrel{d}{\rightarrow} N(0,1) .
$$

We prove Theorem 2 analogously to Theorem 1 . We need some counterparts of Lemmas 3 and 4.

LeMma 6. For arbitrary $i=1, \ldots, k$ we have

$$
\begin{array}{r}
\sqrt{n}\left(\min _{1 \leq j \leq N_{i, n}} Y_{i, j}-x_{i-1}\right) \log \left(\min _{1 \leq j \leq N_{i, n}} Y_{i, j}-x_{i-1}\right) \stackrel{P}{\rightarrow} 0, \\
\sqrt{n}\left(x_{i}-\max _{1 \leq j \leq N_{i, n}} Y_{i, j}\right) \log \left(x_{i}-\max _{1 \leq j \leq N_{i, n}} Y_{i, j}\right) \stackrel{P}{\rightarrow} 0,
\end{array}
$$

Proof. It is easy to show that $n\left(\min _{1 \leq j \leq n} Y_{i, j}-x_{i-1}\right)$ converges in distribution to an exponentially distributed random variable. In the proof of Lemma 3 we have shown that $(1 / \sqrt{n}) \log \left(\min _{1 \leq j \leq n} \underline{Y}_{j}-x_{i-1}\right) \stackrel{P}{\rightarrow} 0$. Hence

$$
n\left(\min _{1 \leq j \leq n} Y_{i, j}-x_{i-1}\right) \frac{1}{\sqrt{n}} \log \left(\min _{1 \leq j \leq n} Y_{i, j}-x_{i-1}\right) \stackrel{P}{\rightarrow} 0 .
$$

Since $N_{i, n}$ is independent of $\underline{Y}_{i}$ and $N_{i, n} \stackrel{P .1}{\longrightarrow} \infty,(22)$ follows from (24). The proof of (23) is quite similar and is omitted.

Lemma 7. Let $X_{i, n}^{*}, i=1, \ldots, k, n \geq 0$, be the random variables defined as follows:

$$
X_{i, n}^{*}= \begin{cases}0 & \text { for } n=0 \\ \frac{\sqrt{n}}{\sqrt{\pi^{2} / 3-3}}\left[\phi^{*}\left(n, \underline{Y}_{i}\right)+\left|I_{i}\right| \log \frac{n}{\left|I_{i}\right|}-\left|I_{i}\right|(1-\gamma)\right] & \text { for } n \geq 1 .\end{cases}
$$


Then

$$
\begin{aligned}
\left(X_{1, N_{1, n}}^{*}, \ldots, X_{k, N_{k, n}}^{*}, \frac{N_{1, p}-n p_{1}}{\sqrt{n}}, \ldots, \frac{N_{k, n}-n p_{k}}{\sqrt{n}}\right) & \\
\stackrel{d}{\rightarrow} & \left(\left|I_{1}\right| X_{1}, \ldots,\left|I_{k}\right| X_{k}, W_{1}, \ldots, W_{k}\right),
\end{aligned}
$$

where $\left(X_{1}, \ldots, X_{k}, W_{1}, \ldots, W_{k}\right)$ is defined by (13) in Lemma 4 .

Proof. Taking into account the remark at the beginning of the proof of Lemma 4, we deduce from Lemma 5 that

$$
\frac{\sqrt{n}}{\sqrt{\pi^{2} / 3-3}}\left(\phi^{*}\left(n, \frac{\underline{Y}_{i}}{\left|I_{i}\right|}\right)+\log n-(1-\gamma)\right) \stackrel{d}{\rightarrow} X_{i} .
$$

After some easy calculations we get

$$
\frac{\sqrt{n}}{\sqrt{\pi^{2} / 3-3}}\left(\phi^{*}\left(n, \underline{Y}_{i}\right)+\left|I_{i}\right| \log \frac{n}{\left|I_{i}\right|}-\left|I_{i}\right|(1-\gamma)\right) \stackrel{d}{\rightarrow}\left|I_{i}\right| X_{i} .
$$

The rest of the proof is similar to the proof of Lemma 4 and is omitted.

Proof of Theorem 2. Similarly to the proof of Theorem 1 the statistic $\phi^{*}(n, \underline{Z})$ can be replaced by $\sum_{i=1}^{k} \phi^{*}\left(N_{i, n}, \underline{Y}_{i}\right)$. Set $D=1 / \sqrt{\pi^{2} / 3-3}$. We have to show that

$$
\frac{D \sqrt{n}\left(S_{n}^{*}+E\left[\frac{\log \left(n f\left(Z_{1}\right)\right)}{f\left(Z_{1}\right)}\right]-(1-\gamma)\right)}{\sqrt{\left(1+D^{2}\right) \operatorname{Var}\left[1 / f\left[Z_{1}\right)\right]+1}} \stackrel{d}{\rightarrow} N(0,1) .
$$

We have the following identity:

$$
\begin{aligned}
D \sqrt{n}\left[\sum_{i=1}^{k} \phi^{*}\left(N_{i, n}, \underline{Y}_{i}\right)+\sum_{i=1}^{k}\left|I_{i}\right| \log \frac{n p_{i}}{\left|I_{i}\right|}-(1-\gamma)\right] \\
=D \sqrt{n}\left[\sum_{i=1}^{k} \phi^{*}\left(N_{i, n}, \underline{Y}_{i}\right)+\sum_{i=1}^{k}\left|I_{i}\right| \log \frac{N_{i, n}}{\left|I_{i}\right|}-(1-\gamma)\right] \\
\quad-D \sum_{i=1}^{k} \frac{\left|I_{i}\right|}{p_{i}} \frac{N_{i, n}-n p_{i}}{\sqrt{n}}-D \sum_{i=1}^{k}\left|I_{i}\right| \sqrt{n} \alpha\left(\frac{N_{i, n}-n p_{i}}{n p_{i}}\right),
\end{aligned}
$$

where $\alpha(x)=\log (1+x)-x$. Similarly to the proof of Theorem 1 we can show that

$$
\sum_{i=1}^{k}\left|I_{i}\right| \sqrt{n} \alpha\left(\frac{N_{i, n}-n p_{i}}{n p_{i}}\right) \stackrel{P}{\rightarrow} 0
$$


Hence Lemma 7 yields that

$$
\begin{aligned}
D \sqrt{n}\left(\sum_{i=1}^{k} \phi^{*}\left(N_{i, n}, \underline{Y}_{i}\right)+E\left[\frac{\log \left(n f\left(Z_{1}\right)\right)}{f\left(Z_{1}\right)}\right]\right. & -(1-\gamma)) \\
& \stackrel{d}{\rightarrow} \sum_{i=1}^{k} \frac{\left|I_{i}\right|}{\sqrt{p_{i}}} X_{i}-D \sum_{i=1}^{k} \frac{\left|I_{i}\right|}{p_{i}} W_{i} .
\end{aligned}
$$

Computing the variance of the right side above, we get the conclusion of Theorem 2.

\section{The consistency of tests based on the Darling and Gebert-} Kale statistics. Let us now consider the problem of testing the hypothesis that a given distribution $F$ is equal to the uniform distribution on $[0,1]$. Using Theorems 1 and 2 we can show that tests based on the statistics $\phi$ and $\phi^{*}$ are consistent if the alternative distributions have a density of type (1). For $\phi$ we have

$$
\begin{aligned}
& \frac{1}{\sqrt{n}}[\phi(n, \underline{Z})+n(\log n+\gamma)] \\
& \quad=\frac{1}{\sqrt{n}}\left[\phi(n, \underline{Z})+n\left(E\left[\log \left(n f\left(Z_{1}\right)\right)\right]+\gamma\right)\right]-\sqrt{n} E\left[\log \left(f\left(Z_{1}\right)\right)\right] .
\end{aligned}
$$

The desired consistency follows from the known fact that $E\left[\log \left(f\left(Z_{1}\right)\right)\right]>$ 0 if the density $f$ is not identically 1 . Similarly for $\phi^{*}$ the consistency is equivalent to $E\left[\log \left(f\left(Z_{1}\right)\right) / f\left(Z_{1}\right)\right]<0$ for $f$ not identically 1 .

\section{References}

[1] D. A. Darling, On a class of problems relating to the random division of an interval, Ann. Math. Statist. 24 (1953), 239-253.

[2] J. R. Gebert and B. K. Kale, Goodness of fit tests based on discriminatory information, Statist. Hefte 3 (1969), 192-200.

[3] L. Weiss, The asymptotic power of certain tests of fit based on sample spacings, Ann. Math. Statist. 28 (1957), 783-786.

[4] - The limiting joint distribution of the largest and smallest sample spacings, ibid. 30 (1959), 590-593.

FRANCISZEK CZEKAEA

MATHEMATICAL INSTITUTE

UNIVERSITY OF WROCEAW

PL. GRUNWALDZKI 2/4

50-384 WROCEAW, POLAND 